\title{
Upper semicontinuity of attractors for nonclassical diffusion equations with arbitrary polynomial growth
}

\author{
Yongqin Xie ${ }^{1}$, Jun $\mathrm{Li}^{1 *}$ (D) and Kaixuan Zhu
}

\section{"Correspondence: \\ ljun931030@163.com \\ 'School of Mathematics and and Statistics, Changsha University of Science and Technology, Changsha, China \\ Full list of author information is available at the end of the article}

\begin{abstract}
In this paper, we mainly investigate upper semicontinuity and regularity of attractors for nonclassical diffusion equations with perturbed parameters $v$ and the nonlinear term $f$ satisfying the polynomial growth of arbitrary order $p-1(p \geq 2)$. We extend the asymptotic a priori estimate method (see (Wang et al. in Appl. Math. Comput. 240:51-61, 2014)) to verify asymptotic compactness and upper semicontinuity of a family of semigroups for autonomous dynamical systems (see Theorems 2.2 and 2.3). By using the new operator decomposition method, we construct asymptotic contractive function and obtain the upper semicontinuity for our problem, which generalizes the results obtained in (Wang et al. in Appl. Math. Comput. 240:51-61, 2014). In particular, the regularity of global attractors is obtained, which extends and improves some results in (Xie et al. in J. Funct. Spaces 2016:5340489, 2016; Xie et al. in Nonlinear Anal. 31:23-37, 2016).
\end{abstract}

Keywords: Nonclassical diffusion equation; Global attractor; Regularity; Upper semicontinuity; Arbitrary polynomial growth

\section{Introduction}

In this paper, we consider the following perturbed nonclassical diffusion equation:

$$
u_{t}-v \Delta u_{t}-\Delta u+f(u)=g \text { in } \Omega \times[0, \infty) .
$$

The problem is supplemented with the boundary condition

$$
\left.u(x, t)\right|_{\partial \Omega}=0 \quad \text { for all } t \geq 0
$$

and the initial condition

$$
\left.u(x, t)\right|_{t=0}=u_{0}(x),
$$

where $\Omega$ is a bounded smooth domain in $\mathbb{R}^{n}(n \geq 3), v \in[0,+\infty)$ is a perturbed parameter, and $g \in L^{2}(\Omega)$ is a given external force term.

(c) The Author(s) 2021. This article is licensed under a Creative Commons Attribution 4.0 International License, which permits use, sharing, adaptation, distribution and reproduction in any medium or format, as long as you give appropriate credit to the original author(s) and the source, provide a link to the Creative Commons licence, and indicate if changes were made. The images or other third party material in this article are included in the article's Creative Commons licence, unless indicated otherwise in a credit line to the material. If material is not included in the article's Creative Commons licence and your intended use is not permitted by statutory regulation or exceeds the permitted use, you will need to obtain permission directly from the copyright holder. To view a copy of this licence, visit http://creativecommons.org/licenses/by/4.0/. 
The nonlinearity $f \in C^{1}$ fulfills $f(0)=0$ and satisfies the following arbitrary-order polynomial growth condition:

$$
\gamma_{1}|s|^{p}-\beta_{1} \leq f(s) s \leq \gamma_{2}|s|^{p}+\beta_{2}, \quad p \geq 2,
$$

and the dissipative condition

$$
f^{\prime}(s) \geq-l,
$$

where $\gamma_{i}, \beta_{i}(i=1,2)$, and $l$ are positive constants. Let $F(s)=\int_{0}^{s} f(v) d v$, then there exist positive constants $\tilde{\gamma}_{i}, \tilde{\beta}_{i}(i=1,2)$ such that

$$
\tilde{\gamma_{1}}|s|^{p}-\tilde{\beta}_{1} \leq F(s) \leq \tilde{\gamma_{2}}|s|^{p}+\tilde{\beta}_{2}
$$

This equation appears as an extension of the usual diffusion equation in fluid mechanics, solid mechanics, and heat conduction theory (see, e.g., [4-7]). Equation (1.1) with a first-order time derivative appearing in the highest-order term is called pseudo-parabolic or Sobolev-Galpern equation [8-10]. In [4], Aifantis proposed a general frame for establishing this equation for certain classes of materials such as polymer and high-viscosity liquids.

Asymptotic behavior similar to Eq. (1.1) has been investigated in many documents during the last years (see, e.g., [2, 3, 11-15] and the references therein).When $v=0$, Eq. (1.1) can be simplified to a usual reaction-diffusion equation, so the dynamical behavior of this equations has been investigated in many documents (see, e.g., [16-19] and the references therein). When $v>0$, if the perturbation parameter $v$ is fixed, many researchers studied the asymptotic behavior of the solutions under different conditions (see, e.g., [2, 3] and the references therein). And the long-time behavior of solutions of Eq. (1.1) has been considered by some researchers (see, e.g., [20, 21] and the references therein).

Therefore, it is natural to examine the limiting behavior of solutions to Eq. (1.1) as $v \rightarrow 0$ (see, e.g., [20-24] and the references therein). For example, in [24], the authors studied the existence of global attractors in $D(A)$ (generated by strong solutions) and their upper semicontinuity in $H_{0}^{1}(\Omega)$ for Eq. (1.1) with subcritical nonlinearity. In [22], upper semicontinuity of pullback attractors in $H_{0}^{1}(\Omega)$ for Eq. (1.1) with subcritical nonlinearity was considered. In [23], upper semicontinuity of attractors in $H^{1}\left(\mathbb{R}^{n}\right)$ for Eq. (1.1) with subcritical nonlinearity was considered. In [20], upper semicontinuity of uniform attractors for Eq. (1.1) was considered, and the nonlinearity satisfies critical exponential growth conditions. In [21], upper semicontinuity of pullback attractors for Eq. (1.1) was considered, and the nonlinearity satisfies critical exponential growth conditions. In [25], with memory lacking instantaneous damping for Eq. (1.1) was considered, and the nonlinearity satisfies critical exponential growth conditions.

In this paper, our main purpose is to consider upper semicontinuity of attractors for Eq. (1.1) with the nonlinearity satisfying arbitrary-order polynomial growth condition, which makes the Sobolev compact embedding no longer valid and brings more difficulties for verifying the corresponding asymptotic compactness of the family of solutions semigroup $\left\{S^{v}(t)\right\}_{t \geq 0}, v \in[0,+\infty)$. In the existing literature, many methods are not applicable to overcome these difficulties (see, e.g., [26-32]). In order to overcome the diffi- 
culty mentioned above, we introduce the asymptotic contractive function method to verify asymptotic compactness of a family of semigroups for autonomous dynamical systems (see Theorem 2.2) by referring to the methods and ideas in [2,3]. Then, by using the new operator decomposition method, we obtain the corresponding asymptotic regularity of the solutions for Eq. (1.1), which ensures the existence of asymptotic contractive function for our problem, which generalizes the results obtained in [1].

For convenience, hereafter let $|u|$ be the modular (or absolute value) of $u$ and $|\cdot|_{p}$ be the norm of $L^{p}(\Omega)(p \geq 1)$. Let $\mathcal{V}=H_{0}^{1}(\Omega)$ and $\|\cdot\|_{0}=|\nabla \cdot|_{2}$ be the norm of $\mathcal{V}$. Denote $A=-\Delta$ with domain $D(A)=H^{2}(\Omega) \cap H_{0}^{1}(\Omega)$. C means any positive constant and $Q(\cdot)$ is a monotonically increasing function on $[0, \infty)$, which may be different from line to line, even in the same line. Let $I \subset[0,+\infty)$ be a bounded closed interval.

The main results of this paper are given in the following two theorems, which will be proved in Sect. 2 and Sect. 3 respectively.

Theorem 1.1 (Global attractors) Let $\Omega \subset \mathbb{R}^{n}$ be a bounded domain with smooth boundary, $v \in I \subset[0,+\infty), f$ satisfy (1.4)-(1.5), and $g \in L^{2}(\Omega)$. Then the semigroup $\left\{S^{\nu}(t)\right\}_{t \geq 0}$, corresponding to (1.1)-(1.3), has a compact global attractor $\mathscr{A}_{\nu}$ in $H_{0}^{1}(\Omega)$ for each and every v. Moreover, for any $v(>0)$ fixed,

$$
\mathscr{A}_{\nu} \subset D(A) \text {. }
$$

This theorem gives the existence and regularity of the family of global attractors.

Theorem 1.2 (Upper semicontinuity) Let $\Omega \subset \mathbb{R}^{n}$ be a bounded domain with smooth boundary, $f$ satisfy (1.4)-(1.6), and $g \in L^{2}(\Omega)$. Let $\left\{\mathscr{A}_{\nu}\right\}$ be the family of global attractors given by Theorem 1.1, and then it satisfies, for every $\nu_{0} \geq 0$,

$$
\lim _{v \rightarrow v_{0}} \operatorname{dist}_{H_{0}^{1}}\left(\mathscr{A}_{v}, \mathscr{A}_{\nu_{0}}\right)=0
$$

where dist $H_{0}^{1}$ denotes the standard Hausdorff semidistance in $H_{0}^{1}(\Omega)$.

It is worth noting that Theorem 1.2 is also interesting in the nonautonomous case (i.e., $g$ is dependent on $t$ ) (see, e.g., [1, 22, 24]). Obviously the results obtained herein are also applicable to considering upper semicontinuity of global attractors for Eq. (1.1) with memory [3] or in unbounded domain [1, 2, 23]. Particularly, the nonlinearity $f$ is assumed to satisfy the polynomial growth of arbitrary order instead of critical nonlinearity (see, e.g., $[20,23])$.

The plan of this paper is as follows. In Sect. 2, we recall some basic concepts and results that are used later. In Sect. 3, by using the ideas in [23], we first verify the asymptotic regularity of the solutions of Eq. (1.1) with (1.2)-(1.3). Then we prove the existence and regularity of global attractors for Eq. (1.1). Finally, we obtain the upper semicontinuity of global attractors for Eq. (1.1) on $H_{0}^{1}(\Omega)$.

\section{Preliminaries}

Definition 2.1 ([2]) Let $X$ be a Banach space and $B$ be a bounded subset of $X, I$ be a parameter interval. We call a function $\varphi(\cdot, \cdot, \cdot, \cdot)$ asymptotic contractive function on $B^{2} \times I^{2}$ 
if there is a contractive function $\phi(\cdot, \cdot, \cdot, \cdot)$ such that, for any $\varepsilon>0$, any sequences $\left\{x_{n}\right\} \subset B$, and $\left\{v_{n}\right\} \subset I$, there exist subsequences $\left\{x_{n_{k}}\right\} \subset\left\{x_{n}\right\}$ and $\left\{v_{n_{k}}\right\} \subset\left\{v_{n}\right\}$ satisfying

$$
\varphi\left(x_{n_{k}}, x_{n_{l}}, v_{n_{k}}, v_{n_{l}}\right) \leq \varepsilon+\phi\left(x_{n_{k}}, x_{n_{l}}, v_{n_{k}}, v_{n_{l}}\right)
$$

and

$$
\lim _{k \rightarrow \infty} \lim _{l \rightarrow \infty} \phi\left(x_{n_{k}}, x_{n_{l}}, v_{n_{k}}, v_{n_{l}}\right)=0 .
$$

We denote the set of all asymptotic contractive functions on $B^{2} \times I^{2}$ by $\mathfrak{E}(B, I)$.

In the following theorem, we present a new method(or technique) to verify the relative compactness of a two-parameter sequence for the family of semigroups generated by evolutionary equations, which will be used in our later discussion.

Theorem 2.2 Let $\left(X,\|\cdot\|_{X}\right)$ be a Banach space, $B$ be a bounded subset of $X$, and I be a parameter interval. Assume further that $\left\{S^{v}(t)\right\}_{t \geq 0}, v \in I$, is a family of semigroups on $X$ which satisfy the following conditions:

(i) $\left\{S^{v}(t)\right\}_{t \geq 0}, v \in I$, has a bounded uniformly (w.r.t. $v$ ) absorbing set $B_{0}$ in $X$;

(ii) For any $\varepsilon>0$ and $x, y \in B_{0}, \nu_{1}, v_{2} \in I$, there exist $T=T\left(B_{0}, \varepsilon\right)>0$ and $\varphi_{T} \in \mathfrak{E}\left(B_{0}, I\right)$ such that

$$
\left\|S^{\nu_{1}}(T) x-S^{\nu_{2}}(T) y\right\|_{X} \leq \varepsilon+\varphi_{T}\left(x, y, \nu_{1}, \nu_{2}\right) .
$$

Then the sequence $\left\{S^{v_{n}}\left(t_{n}\right) x_{n}\right\}$ is precompact in $X$ for any $\left\{x_{n}\right\} \subset B,\left\{v_{n}\right\} \subset I$, and $t_{n} \stackrel{n \rightarrow \infty}{\longrightarrow \infty}$, where $\varphi_{T}$ depends on $T$.

Proof Let $\left\{x_{n}\right\} \subset B \subset X,\left\{v_{n}\right\} \subset I \subset[0,+\infty)$, and $t_{n} \rightarrow+\infty$ as $n \rightarrow \infty$. By (i), for every $v \in I$, there is $t_{N}>0$ such that $S^{v}\left(t_{N}\right) x \in B_{0}$. For all $t_{n}>t_{N}, v \in I$, and $x \in B$, it follows that

$$
S^{\nu}\left(t_{n}\right) x=S^{\nu}\left(t_{n}-t_{N}\right) \cdot S^{\nu}\left(t_{N}\right) x=S^{\nu}\left(t_{n}-t_{N}\right) y=S^{\nu}\left(t_{n}^{\prime}\right) y,
$$

here $y=S^{v}\left(t_{N}\right) x \in B_{0}, t_{n}^{\prime}=t_{n}-t_{N}>0$, and $v \in I$. Then we just need to consider this case as $\left\{x_{n}\right\} \subset B_{0}$.

The following work is to prove the existence of a Cauchy subsequence of $\left\{S^{v_{n}}\left(t_{n}\right) x_{n}\right\}$ by the diagonal method.

Taking $\varepsilon_{m}>0$ with $\varepsilon_{m} \rightarrow 0$ as $m \rightarrow \infty$, for $\varepsilon_{1}$, then there exist $T_{1}=T_{1}\left(\varepsilon_{1}\right)$ and $\varphi_{T_{1}} \in$ $\mathfrak{E}\left(B_{0}, I\right)$ such that

$$
\left\|S^{\nu_{1}}\left(T_{1}\right) x-S^{\nu_{2}}\left(T_{1}\right) y\right\|_{X} \leq \varepsilon_{1}+\varphi_{T_{1}}\left(x, y, \nu_{1}, \nu_{2}\right) \quad \text { for any } x, y \in B_{0}, \nu_{1}, \nu_{2} \in I \text {. }
$$

Since $t_{n} \rightarrow+\infty$, for $T_{1}$ fixed, we assume that $t_{n} \gg T_{1}$ is so large that $S^{v_{n}}\left(t_{n}-T_{1}\right) x_{n} \in B_{0}$ for each $n \geq N$ and $v_{n} \in I$.

Let $y_{k}=S^{v_{k}}\left(t_{k}-T_{1}\right) x_{k}$, then we have

$$
\begin{aligned}
& \left\|S^{\nu_{n}}\left(t_{n}\right) x_{n}-S^{\nu_{m}}\left(t_{m}\right) x_{m}\right\|_{X} \\
& \quad=\left\|S^{\nu_{n}}\left(T_{1}\right) \cdot S^{\nu_{n}}\left(t_{n}-T_{1}\right) x_{n}-S^{\nu_{m}}\left(T_{1}\right) \cdot S^{\nu_{m}}\left(t_{m}-T_{1}\right) x_{m}\right\|_{X}
\end{aligned}
$$




$$
\begin{aligned}
& =\left\|S^{v_{n}}\left(T_{1}\right) y_{n}-S^{v_{m}}\left(T_{1}\right) y_{m}\right\|_{X} \\
& \leq \varepsilon_{1}+\varphi_{T_{1}}\left(y_{n}, y_{m}, v_{n}, v_{m}\right) .
\end{aligned}
$$

Due to the definition of $\mathfrak{E}\left(B_{0}, I\right)$ and $\varphi_{T_{1}} \in \mathfrak{E}\left(B_{0}, I\right)$, we know that there exist a contractive function $\phi_{T_{1}}$ and a subsequence $\left\{\left(y_{n_{k}}^{(1)}, v_{n_{k}}^{(1)}\right)\right\}$ of $\left\{\left(y_{n}, v_{n}\right)\right\}$ such that

$$
\varphi_{T_{1}}\left(y_{n_{k}}^{(1)}, y_{n_{l}}^{(1)}, v_{n_{k}}^{(1)}, v_{n_{l}}^{(1)}\right) \leq \varepsilon_{1}+\phi_{T_{1}}\left(y_{n_{k}}^{(1)}, y_{n_{l}}^{(1)}, v_{n_{k}}^{(1)}, v_{n_{l}}^{(1)}\right)
$$

and

$$
\lim _{k \rightarrow \infty} \lim _{l \rightarrow \infty} \phi_{T_{1}}\left(y_{n_{k}}^{(1)}, y_{n_{l}}^{(1)}, v_{n_{k}}^{(1)}, v_{n_{l}}^{(1)}\right)=0 .
$$

Combined with (2.2) and (2.3), we have

$$
\begin{aligned}
& \lim _{k \rightarrow \infty} \sup _{p \in \mathbb{N}}\left\|S^{v_{n_{k+p}}^{(1)}}\left(t_{n_{k+p}}^{(1)}\right) x_{n_{k+p}}^{(1)}-S^{v_{n_{k}}^{(1)}}\left(t_{n_{k}}^{(1)}\right) x_{n_{k}}^{(1)}\right\|_{X} \\
& \leq \lim _{k \rightarrow \infty} \sup _{p \in \mathbb{N}} \limsup _{l \rightarrow \infty}\left\|S^{v_{n_{k+p}}^{(1)}}\left(t_{n_{k+p}}^{(1)}\right) x_{n_{k+p}}^{(1)}-S^{v_{n_{l}}^{(1)}}\left(t_{n_{l}}^{(1)}, \tau\right) x_{n_{l}}^{(1)}\right\|_{X} \\
& \quad+\limsup _{k \rightarrow \infty} \limsup _{l \rightarrow \infty}\left\|S^{v_{n_{k}}^{(1)}}\left(t_{n_{k}}^{(1)}\right) x_{n_{k}}^{(1)}-S^{v_{n_{l}}^{(1)}}\left(t_{n_{l}}^{(1)}\right) x_{n_{l}}^{(1)}\right\|_{X} \\
& \leq \varepsilon_{1}+\lim _{k \rightarrow \infty} \sup _{p \in \mathbb{N}} \lim _{l \rightarrow \infty} \varphi_{T_{1}}\left(y_{n_{k+p}}^{(1)}, y_{n_{l}}^{(1)}, v_{n_{k+p}}^{(1)}, v_{n_{l}}^{(1)}\right) \\
& \quad+\varepsilon_{1}+\lim _{k \rightarrow \infty} \lim _{l \rightarrow \infty} \varphi_{T_{1}}\left(y_{n_{k}}^{(1)}, y_{n_{l}}^{(1)}, v_{n_{k}}^{(1)}, v_{n_{l}}^{(1)}\right) \\
& \leq 5 \varepsilon_{1} .
\end{aligned}
$$

Therefore, there exists $N_{1}$ such that

$$
\left\|S^{v_{n_{k}}^{(1)}}\left(t_{n_{k}}^{(1)}\right) x_{n_{k}}^{(1)}-S^{v_{n_{l}}^{(1)}}\left(t_{n_{l}}^{(1)}\right) x_{n_{l}}^{(1)}\right\|_{X} \leq 6 \varepsilon_{1} \quad \text { for all } k, l \geq N_{1}
$$

By induction, for each $m \geq 1$, there exists a subsequence $\left\{S^{v_{n_{k}}^{(m+1)}}\left(t_{n_{k}}^{(m+1)}\right) x_{n_{k}}^{(m+1)}\right\}$ of $\left\{S^{v_{n_{k}}^{(m)}}\left(t_{n_{k}}^{(m)}\right) x_{n_{k}}^{(m)}\right\}$ and certain $N_{m+1}$ such that, for all $k, l \geq N_{m+1}$,

$$
\left\|S^{\nu_{n_{k}}^{(m+1)}}\left(t_{n_{k}}^{(m+1)}\right) x_{n_{k}}^{(m+1)}-S^{v_{n_{l}}^{(m+1)}}\left(t_{n_{l}}^{(m+1)}\right) x_{n_{l}}^{(m+1)}\right\|_{X} \leq 6 \varepsilon_{m+1}
$$

holds. Next, we consider the diagonal subsequence $\left\{S^{v_{n_{k}}^{(k)}}\left(t_{n_{k}}^{(k)}\right) x_{n_{k}}^{(k)}\right\}$. Since for each $m \in \mathbb{N}$, $\left\{S^{\left(v_{n_{k}}^{(k)}\right.}\left(t_{n_{k}}^{(k)}\right) x_{n_{k}}^{(k)}\right\}$ is a subsequence of $\left\{S^{v_{n_{k}}^{(m)}}\left(t_{n_{k}}^{(m)}\right) x_{n_{k}}^{(m)}\right\}$, then

$$
\left\|S^{v_{n_{k}}^{(k)}}\left(t_{n_{k}}^{(k)}\right) x_{n_{k}}^{(k)}-S^{v_{n_{l}}^{(l)}}\left(t_{n_{l}}^{(l)}\right) x_{n_{l}}^{(l)}\right\|_{X} \leq 6 \varepsilon_{m} \quad \text { for all } k, l \geq \max \left\{m, N_{m}\right\}
$$

which, combined with $\varepsilon_{m} \rightarrow 0$ as $m \rightarrow \infty$, implies that $\left\{S^{v_{n_{k}}^{(k)}}\left(t_{n_{k}}^{(k)}\right) x_{n_{k}}^{(k)}\right\}$ is a Cauchy sequence in $X$. This shows that $\left\{S^{v_{n}}\left(t_{n}\right) x_{n}\right\}$ is precompact in $X$. Then the proof is complete.

For more details of the standard theory of global attractors, we recommend the readers to refer to [33]. Now, we present the following theorem to verify upper semicontinuity of global attractors in autonomous dynamical systems. 
Theorem 2.3 ([20]) Let $X, Y$ be two Banach spaces with $X \hookrightarrow Y$ and $I \subset[0,+\infty)$ be a bounded closed interval. Assume that, for any $v \in I$, the family of semigroups $\left\{S^{v}(t)\right\}_{t \geq 0}$ possesses a bounded uniformly (w.r.t. $v$ ) absorbing set $B_{0}$ in X. If the following assumptions hold true:

(1) $\left\{S^{v}(t)\right\}_{t \geq 0}$ has a global attractor $\mathscr{A}_{v}$ in $X$ for every $v \in I$;

(2) For any $\varepsilon>0, x, y \in B_{0}, v_{1}, v_{2} \in I$, there exist $T=T\left(B_{0}, \varepsilon\right)>0$ and $\varphi_{T} \in \mathfrak{E}\left(B_{0}, I\right)$ such that

$$
\left\|S^{\nu_{1}}(T) x-S^{\nu_{2}}(T) y\right\|_{X} \leq \varepsilon+\varphi_{T}\left(x, y, v_{1}, \nu_{2}\right)
$$

(3) For any $t \geq 0$, any sequences $\left\{v_{n}\right\} \subset I$ and $\left\{x_{n}\right\} \subset X$ with $v_{n} \stackrel{n \rightarrow \infty}{\longrightarrow} v_{0}$ and $x_{n} \stackrel{n \rightarrow \infty}{\longrightarrow} x_{0}$ in $X$,

$$
S^{\nu_{n}}(t) x_{n} \rightarrow S^{\nu_{0}}(t) x_{0}, \quad \text { in } Y
$$

Then

$$
\lim _{v \rightarrow v_{0}} \operatorname{dist}_{X}\left(\mathscr{A}_{v}, \mathscr{A}_{v_{0}}\right)=0
$$

Lemma 2.4 ([33]) Let $X \subset \subset H \subset Y$ be Banach spaces with $X$ reflexive. Suppose that $u_{n}$ is a sequence which is uniformly bounded in $L^{2}(0, T ; X)$ and $d u_{n} / d t$ is uniformly bounded in $L^{p}(0, T ; Y)$ for some $p>1$. Then there exists a subsequence of $u_{n}$ that converges strongly in $L^{2}(0, T ; H)$.

\section{Global attractors in $H_{0}^{1}(\Omega)$}

\subsection{A priori estimates}

We start with the following general existence and uniqueness of solutions for the nonclassical diffusion equations which can be obtained by the Galerkin approximation methods (see [33] for more details), here we only formulate the results.

Lemma 3.1 Provided that $f$ satisfies (1.4)-(1.5). Then, for any initial data $u_{0} \in \mathcal{V}$ and any $T>0$, there exists a unique solution $u(t)$ for problem (1.1)-(1.3). Moreover, we have the following Lipschitz continuity: For any $u_{0}^{i} \in \mathcal{V}$, denote by $u_{i}(t)(i=1,2)$ the corresponding solutions of Eq. (1.1), then for all $0 \leq t \leq T$

$$
\left|u_{1}(t)-u_{2}(t)\right|_{2}^{2}+v\left\|u_{1}(t)-u_{2}(t)\right\|_{0}^{2} \leq C e^{\kappa(T-\tau)}\left(\left\|u_{0}^{1}-u_{0}^{2}\right\|_{0}^{2}\right)
$$

where $\kappa>0$ is a constant.

By Lemma 3.1, we can define a semigroup $S^{\nu}(t)$ in $\mathcal{V}$ as follows:

$$
\begin{aligned}
& S^{\nu}(t): \mathbb{R}^{+} \rightarrow \mathcal{V}, \\
& t \rightarrow u(t)=S^{\nu}(t) u_{0},
\end{aligned}
$$

and $\left\{S^{\nu}(t)\right\}_{t \geq 0}$ is a continuous semigroup on $\mathcal{V}$. 
Lemma 3.2 ([2]) Let (1.4)-(1.5) hold, B be any bounded subset in $\mathcal{V}$. Then there exist positive constants $\alpha$ and $k_{0}$, which are independent of $v$, such that

$$
|u|_{2}^{2}+v\|u\|_{0}^{2} \leq Q\left(\left\|u_{0}\right\|_{0}\right) e^{-\alpha t}+k_{0}
$$

and

$$
\int_{t}^{t+1}\left(\|u(s)\|_{0}^{2}+|u(s)|_{p}^{p}\right) d s \leq Q\left(\left\|u_{0}\right\|_{0}\right) e^{-\alpha t}+k_{0}
$$

hold for any $u_{0} \in B$ and $t \geq 0$.

Lemma 3.3 Let (1.4)-(1.5) hold, then there exists positive $\rho_{0}$, which depends on $\|g\|_{2}$ and does not rely on $v$, such that for any bounded subset $B \subset \mathcal{V}$, there is $T_{0}=T_{0}(\|B\| \mathcal{V})$ such that

$$
\left\|S^{\nu}(t) u_{0}\right\|_{0}^{2}+\left|S^{\nu}(t) u_{0}\right|_{p}^{p} \leq \rho_{0} \quad \text { for all } t \geq T_{0} \text { and all } u_{0} \in B
$$

Proof Multiplying (1.1) by $u_{t}+u$ and then integrating over $\Omega$, we obtain that

$$
\begin{aligned}
& \frac{d}{d t}\left(\frac{1}{2}|u|_{2}^{2}+\frac{1+v}{2}\|u\|_{0}^{2}+\int_{\Omega} F(u)-\int_{\Omega} g u\right)+\left|u_{t}\right|_{2}^{2}+v\left\|u_{t}\right\|_{0}^{2}+\|u\|_{0}^{2} \\
& \quad=-\langle f(u), u\rangle+\langle g, u\rangle .
\end{aligned}
$$

Observe that

$$
\langle f(u), u\rangle=\int_{\Omega} f(u) u \geq \gamma_{1}|u|_{p}^{p}-\beta_{1} \operatorname{mes}(\Omega) .
$$

Using the Hölder inequality, we have

$$
\langle g, u\rangle \leq \frac{1}{\sqrt{\lambda_{1}}}|g|_{2}\|u\|_{0} \leq \frac{1}{2 \lambda_{1}}|g|_{2}^{2}+\frac{1}{2}\|u\|_{0}^{2},
$$

where $\lambda_{1}$ is the first eigenvalue of $-\Delta$ on $H_{0}^{1}(\Omega)$.

Let

$$
E(t)=\frac{1}{2}|u|_{2}^{2}+\frac{1+v}{2}\|u\|_{0}^{2}+\int_{\Omega} F(u)-\int_{\Omega} g u .
$$

Combining with (3.6) and (3.7), then (3.5) can be reformulated as follows:

$$
\begin{aligned}
& \frac{d}{d t} E(t)+\frac{1}{2}\|u\|_{0}^{2}+\gamma_{1}|u|_{p}^{p}+\frac{1}{2}\left|u_{t}\right|_{2}^{2}+\frac{v}{2}\left\|u_{t}\right\|_{0}^{2} \\
& \quad \leq \beta_{1} \operatorname{mes}(\Omega)+\frac{1}{2 \lambda_{1}}|g|_{2}^{2} .
\end{aligned}
$$

By (1.6), we obtain that

$$
\begin{aligned}
E(t) & \leq|u|_{2}^{2}+\frac{1+v}{2}\|u\|_{0}^{2}+\tilde{\gamma}_{2}|u|_{p}^{p}+\tilde{\beta}_{2} \operatorname{mes}(\Omega)+\frac{1}{2}|g|_{2}^{2} \\
& \leq \alpha_{0}\left(|u|_{2}^{2}+\|u\|_{0}^{2}+|u|_{p}^{p}\right)+\tilde{\beta}_{2} \operatorname{mes}(\Omega)+\frac{1}{2}|g|_{2}^{2},
\end{aligned}
$$


where $\alpha_{0}=\max \left\{1, \frac{1+v}{2}, \tilde{\gamma}_{2}\right\} \geq 1$, and

$$
\begin{aligned}
E(t) & \geq \frac{1}{4}|u|_{2}^{2}+\frac{1+v}{2}\|u\|_{0}^{2}+\tilde{\gamma}_{1}|u|_{p}^{p}-\tilde{\beta}_{1} \operatorname{mes}(\Omega)-|g|_{2}^{2} \\
& \geq \beta_{0}\left(|u|_{2}^{2}+\|u\|_{0}^{2}+|u|_{p}^{p}\right)-\tilde{\beta}_{1} \operatorname{mes}(\Omega)-|g|_{2}^{2},
\end{aligned}
$$

where $\beta_{0}=\min \left\{\frac{1}{4}, \tilde{\gamma}_{1}\right\}$ is independent of $\nu$.

According to (3.9), we get

$$
\frac{d}{d t} E(t)+\alpha_{1} E(t) \leq C\left(1+|g|_{2}^{2}\right)
$$

where $\alpha_{1}=\frac{\lambda_{1} \min \left\{\frac{1}{2}, \gamma_{1}\right\}}{\alpha_{0}\left(1+\lambda_{1}\right)}$.

By Lemma 3.2, for any $0<\delta<1$, there is $t^{\star} \in(0, \delta)$ such that

$$
\left|u\left(t^{\star}\right)\right|_{2}^{2}+\left\|u\left(t^{\star}\right)\right\|_{0}^{2}+\left|u\left(t^{\star}\right)\right|_{p}^{p} \leq Q\left(\left\|u_{0}\right\|_{0}\right)+k_{0}
$$

Applying Gronwall's lemma to (3.11), we obtain that

$$
E(t+\delta) \leq e^{-\alpha_{1}\left(t+\delta-t^{\star}\right)} E\left(t^{\star}\right)+C\left(1+|g|_{2}^{2}\right)
$$

holds for any $t>0$.

Thanks to (3.9) and (3.10), we estimate that

$$
\begin{aligned}
& |u(t+\delta)|_{2}^{2}+\|u(t+\delta)\|_{0}^{2}+|u(t+\delta)|_{p}^{p} \\
& \quad \leq Q\left(\left\|u_{0}\right\|_{0}\right) e^{-\alpha_{0} t}+\frac{\alpha_{0}}{\beta_{0}} k_{0}+C\left(1+|g|_{2}^{2}\right) .
\end{aligned}
$$

Setting

$$
\rho_{0}=\frac{2 \alpha_{0}}{\beta_{0}} k_{0}+2 C\left(1+|g|_{2}^{2}\right), \quad T_{0}=1+\frac{1}{\alpha_{0}} \ln \frac{2 Q\left(\left\|u_{0}\right\|_{0}\right)}{\rho_{0}},
$$

we get

$$
|u(t)|_{2}^{2}+\|u(t)\|_{0}^{2}+|u(t)|_{p}^{p} \leq \rho_{0}
$$

for all $t \geq T_{0}$. The proof is complete.

Combining with (3.1) and taking $\delta \rightarrow 0$ for (3.14), we know that, for any $v \in I, S^{v}$ maps the bounded set of $\mathcal{V}$ into a bounded set for all $t \geq 0$, and there is the following corollary.

Corollary 3.4 Let (1.4)-(1.5) hold, then for any bounded (in $\mathcal{V})$ subset $B$ and any $u_{0} \in B$, the following estimate

$$
|u(t)|_{2}^{2}+\|u(t)\|_{0}^{2}+|u(t)|_{p}^{p} \leq Q\left(\left\|u_{0}\right\|_{0}\right) e^{-\alpha_{0} t}+\frac{1}{2} \rho_{0}
$$

holds for any $t>0$. 
Lemma 3.5 (Bounded uniformly absorbing set) Let (1.4)-(1.5) hold and $g \in L^{2}(\Omega)$. There exists a bounded uniformly (w.r.t. $v \in I$ ) absorbing set $B_{0} \subset \mathcal{V}$ for the semigroup $\left\{S^{v}(t) \mid v \in\right.$ $[0,+\infty)\}_{t \geq 0}$ associated with Eq. (1.1), that is, for any bounded subset $B \subset \mathcal{V}$ and $v \in I$, there exists $T_{0}=T_{0}(B) \geq 0$ such that

$$
\bigcup_{\nu \geq 0} S^{\nu}(t) B \subset B_{0}
$$

holds for all $t \geq T_{0}$.

For brevity, later in this article, let $B_{0}$ be the bounded uniformly (w.r.t. $v$ ) absorbing set obtained in Lemma 3.3, i.e.,

$$
B_{0}=\left\{u \in \mathcal{V}:|u|_{2}^{2}+\|u\|_{0}^{2}+|u|_{p}^{p} \leq \rho_{0}\right\}
$$

Lemma 3.6 Let (1.4)-(1.5) hold and $B$ be any bounded subset $B \subset \mathcal{V}$. There is a positive constant $\mathcal{K}_{0}$; for any $u_{0} \in B$, the following estimate

$$
\int_{0}^{+\infty}\left(\left|u_{t}(s)\right|_{2}^{2}+v\left\|u_{t}(s)\right\|_{0}^{2}\right) d s \leq \mathcal{K}_{0}
$$

holds for any $t \geq 0$.

Proof Multiplying (1.1) by $u_{t}$ and integrating over $\Omega$, we have

$$
\frac{d}{d t}\left(\frac{1}{2}\|u\|_{0}^{2}+\int_{\Omega} F(u)+\int_{\Omega} g u\right)+\left|u_{t}\right|_{2}^{2}+v\left\|u_{t}\right\|_{0}^{2}=0 .
$$

Set

$$
H(t)=\frac{1}{2}\|u(t)\|_{0}^{2}+\int_{\Omega} F(u(t))+\int_{\Omega} g u(t) .
$$

For any $t \geq 0$, we integrate (3.17) to $t$ from 0 to $t$, then we have

$$
H(t)+\int_{0}^{t}\left[\left|u_{t}(s)\right|_{2}^{2}+v\left\|u_{t}(s)\right\|_{0}^{2}\right] d s=H(0)
$$

According to Lemma 3.5, Corollary 3.4, and (3.9)-(3.10), we know that $H(0)$ is bounded and $H(t) \geq-\tilde{\beta}_{1} \operatorname{mes}(\Omega)-|g|_{2}|u(t)|_{2}$, then there exists a positive constant $\mathcal{K}_{0}$ independent of $v$ such that the conclusion is true.

Lemma 3.7 There is a positive constant $\mathcal{K}_{1}$; for any $t>0$, the following estimate

$$
\left|u_{t}(t)\right|_{2}^{2}+v\left\|u_{t}(t)\right\|_{0}^{2}+\int_{0}^{+\infty}\left\|u_{t}(s)\right\|_{0}^{2} d s \leq \mathcal{K}_{1}
$$

holds. 
Proof Differentiating about $t$ for Eq. (1.1), we obtain

$$
u_{t t}-\Delta u_{t}-v \Delta u_{t t}+f^{\prime}(u) u_{t}=0 .
$$

Multiplying (3.19) by $u_{t}$ and then integrating over $\Omega$ leads to

$$
\frac{d}{d t}\left(\left|u_{t}\right|_{2}^{2}+v\left\|u_{t}\right\|_{0}^{2}\right)+\left\|u_{t}\right\|_{0}^{2} \leq l\left|u_{t}\right|_{2}^{2}
$$

By Lemma 3.6, for any $0<\delta<1$, there is $s^{\star} \in(0, \delta)$ such that

$$
\left|u_{t}\left(s^{\star}\right)\right|_{2}^{2}+v\left\|u_{t}\left(s^{\star}\right)\right\|_{0}^{2} \leq \mathcal{K}_{0} .
$$

Now, we integrate (3.20) about $t$ from $s^{\star}$ to $t+\delta(t>0)$, we obtain

$$
\left|u_{t}(t+\delta)\right|_{2}^{2}+v\left\|u_{t}(t+\delta)\right\|_{0}^{2} \leq\left|u_{t}\left(s^{\star}\right)\right|_{2}^{2}+v\left\|u_{t}\left(s^{\star}\right)\right\|_{0}^{2}+l \int_{0}^{t}\left|u_{t}(s)\right|_{2}^{2} d s
$$

Combine with Lemma 3.6 and Corollary 3.4, and let $\delta \rightarrow 0$, then for any $t>0$ it follows that

$$
\left|u_{t}(t)\right|_{2}^{2}+v\left\|u_{t}(t)\right\|_{2}^{2} \leq \mathcal{K}_{0}+l\left(Q\left(\left\|u_{0}\right\|_{0}\right)+\rho_{0}\right)
$$

Integrate (3.20) about $t$ from $s$ to $t$ again, and let $t \rightarrow \infty, s \rightarrow 0^{+}$, it gets

$$
\int_{0}^{+\infty}\left\|u_{t}(s)\right\|_{0}^{2} d s \leq \mathcal{K}_{0}+l\left(Q\left(\left\|u_{0}\right\|_{0}\right)+\rho_{0}\right)
$$

Setting

$$
\mathcal{K}_{1}=2 \mathcal{K}_{0}+3 l\left(Q\left(\left\|u_{0}\right\|_{0}\right)+\rho_{0}\right)
$$

gives that

$$
\left|u_{t}(t)\right|_{2}^{2}+v\left\|u_{t}(t)\right\|_{0}^{2}+\int_{0}^{+\infty}\left\|u_{t}(s)\right\|_{0}^{2} d s \leq \mathcal{K}_{1}
$$

holds for any $t>0$.

\subsection{The asymptotic regularity}

In the following, we prove the asymptotic regularity of solutions for system (1.1) with initial-boundary conditions(1.2)-(1.3) in $\mathcal{V}$ by using a new decomposition method (or technique).

In order to obtain the asymptotic regularity estimates later, we decompose the solution $S^{v}(t) u_{0}=u(t)$ into the following sum:

$$
S^{v}(t) u_{0}=S_{1}^{v}(t) u_{0}+K^{v}(t) u_{0},
$$


where $S_{1}^{v}(t) u_{0}=v(t)$ and $K^{v}(t) u_{0}=\omega(t)$ are solutions of the following equations respectively:

$$
\left\{\begin{array}{l}
v_{t}-\Delta v-v \Delta v_{t}+f(u)-f(\omega)+\mu v=0 \\
v(0)=u_{0},\left.\quad v\right|_{\partial \Omega}=0
\end{array}\right.
$$

and

$$
\left\{\begin{array}{l}
\omega_{t}-\Delta \omega-v \Delta \omega_{t}+f(\omega)+\mu \omega=g+\mu u, \\
\omega(0)=0,\left.\quad \omega\right|_{\partial \Omega}=0,
\end{array}\right.
$$

where the constant $\mu \geq 2 l$ and $l$ are from (1.5).

Remark 3.8 It is easy to verify the existence and uniqueness of the decomposition (3.24) corresponding to (3.25) and (3.26).

In fact, $u$ is the unique solution of Eq. (1.1) with (1.2)-(1.3), thus $g+\mu u \in L^{2}(\Omega)$ is known. The existence and uniqueness of solutions $\omega$ corresponding to Eq. (3.26) can be obtained by the Galerkin approximation method (see [33]). By the superposition principle of solutions of partial differential equations, the existence and uniqueness of solutions $v$ for Eq. (3.25) can be proved.

We use a priori estimate to get the asymptotic regularity of solutions for Eq. (1.1) with (1.2)-(1.3), which are the basis of our analysis. The proof is similar to [2], but the estimate about $\|v\|_{0}$ is not mentioned, only $v\|v\|_{0}$.

Lemma 3.9 Letf satisfy (1.4)-(1.6) and $B_{0}$ be the bounded absorbing set (see Lemma 3.5). Assume that $K^{v}(t) u_{0}=\omega(t)$ is the solutions of $(3.26)$ with $u_{0} \in B_{0}$. Then there exists a positive constant $\rho_{1}$ which depends only on $|g|_{2}$ but does not rely on $v$ such that

$$
\|\omega(t)\|_{0}^{2}+v\|\omega(t)\|_{1}^{2} \leq \rho_{1}
$$

and

$$
\int_{t}^{t+1}\|\omega(s)\|_{1}^{2} d s \leq \rho_{1}
$$

hold for all $t>0$.

Proof Multiplying (3.26) by $-\Delta \omega(t)$ and integrating in $\Omega$, we have

$$
\frac{d}{d t}\left(\|\omega\|_{0}^{2}+v\|\omega\|_{1}^{2}\right)+(\mu-l)\|\omega\|_{0}^{2}+\|\omega\|_{1}^{2} \leq \frac{\mu^{2}}{\mu-l}\|u\|_{0}^{2}+|g|_{2}^{2}
$$

Let $\alpha_{2}=\min \left\{\mu-l, \frac{1}{v}\right\}$ as $v>0$, or else $\alpha_{2}=\mu-l$, then we can rewrite (3.27) as follows:

$$
\frac{d}{d t}\left(\|\omega\|_{0}^{2}+v\|\omega\|_{1}^{2}\right)+\alpha_{2}\left(\|\omega\|_{0}^{2}+v\|\omega\|_{1}^{2}\right) \leq \frac{\mu^{2}}{\mu-l}\|u\|_{0}^{2}+|g|_{2}^{2} .
$$


Applying Gronwall's inequality and Corollary 3.4, we obtain

$$
\|\omega(t)\|_{0}^{2}+v\|\omega(t)\|_{1}^{2} \leq \frac{1}{\alpha_{2}}\left(|g|_{2}^{2}+\frac{\mu^{2}}{\mu-l} Q\left(\rho_{0}\right)\right),
$$

where the initial data $(\omega(0)=0)$ is used.

Now integrating (3.27) between $t$ and $t+1$, we get

$$
\int_{t}^{t+1}\|\omega(s)\|_{1}^{2} d s \leq \frac{\mu^{2}}{\mu-l} \int_{t}^{t+1}\|u(s)\|_{0}^{2} d s+|g|_{2}^{2}+\|\omega(t)\|_{0}^{2}+v\|\omega(t)\|_{1}^{2} .
$$

Set

$$
\rho_{1}=\frac{1+\alpha_{2}}{\alpha_{2}}\left(|g|_{2}^{2}+\frac{\mu^{2}}{\mu-l} Q\left(\rho_{0}\right)\right) .
$$

From (3.28) and (3.29) it follows that

$$
\|\omega(t)\|_{0}^{2}+v\|\omega(t)\|_{1}^{2} \leq \rho_{1}
$$

and

$$
\int_{t}^{t+1}\|\omega(s)\|_{1}^{2} d s \leq \rho_{1}
$$

hold for all $t>0$.

Corollary 3.10 There is a positive constant $\mathcal{K}_{2}$, the following estimate

$$
\left|\omega_{t}(t)\right|_{2}^{2}+v\left\|\omega_{t}(t)\right\|_{0}^{2}+\int_{0}^{+\infty}\left\|\omega_{t}(s)\right\|_{0}^{2} d s \leq \mathcal{K}_{2}
$$

holds for all $t>0$.

Lemma 3.11 Letf satisfy (1.4)-(1.5) and B be any bounded set of $\mathcal{V}$. Assume that $S_{1}^{v}(t) u_{0}=$ $v(t)$ is the solutions of (3.25) with the initial data $v(0)=u_{0} \in B$. Then

$$
\lim _{t \rightarrow \infty}\left(|v(t)|_{2}^{2}+\|v(t)\|_{0}^{2}\right)=0
$$

holds for any $v \in I$.

Proof Multiplying (3.25) by $v(t)$ and integrating in $\Omega$, we have

$$
\frac{d}{d t}\left(|v|_{2}^{2}+v\|v\|_{0}^{2}\right)+2\|v\|_{0}^{2}+2(\mu-l)|v|_{2}^{2} \leq 0
$$

Recall

$$
\alpha_{3}= \begin{cases}\frac{1}{v}, & v \geq \frac{1}{\mu-l}, \\ \mu-l, & v<\frac{1}{\mu-l} .\end{cases}
$$


Rewrite (3.32) as follows:

$$
\frac{d}{d t}\left(|v|_{2}^{2}+v\|v\|_{0}^{2}\right)+\alpha_{3}\left(|v|_{2}^{2}+v\|v\|_{0}^{2}\right) \leq 0 .
$$

Applying Gronwall's inequality, we obtain

$$
|v(t)|_{2}^{2}+v\|v(t)\|_{0}^{2} \leq Q\left(\left\|u_{0}\right\|_{0}\right) e^{-\alpha_{3} t}
$$

Now, integrating (3.32) between 0 and $\infty$, we get

$$
\int_{0}^{\infty}\|v(s)\|_{0}^{2} d s \leq 2\left(\left|u_{0}\right|_{2}^{2}+v\left\|u_{0}\right\|_{0}^{2}\right)
$$

Furthermore, $\|v(t)\|_{0}^{2} \leq 2\left(\|u\|_{0}^{2}+\|\omega\|_{0}^{2}\right)$ is boundary for any $t \geq 0$. Hence, it follows that

$$
\lim _{t \rightarrow \infty}\|v(t)\|_{0}^{2}=0
$$

and by combining with (3.33) we get

$$
\lim _{t \rightarrow \infty}\left(|v(t)|_{2}^{2}+\|v(t)\|_{0}^{2}\right)=0
$$

holds for all $v \in I$.

Proof of Theorem 1.1 Let $u_{i}(t)=S^{\nu}(t) u_{0}^{i}(i=1,2)$ be the solutions to Eq. (1.1) with the parameters $v$ and the initial data $u_{0}^{i}(x) \in B(i=1,2)$ respectively. By (3.24),

$$
u_{i}(t)=S^{\nu}(t) u_{0}^{i}=S_{1}^{v}(t) u_{0}^{i}+K^{v}(t) u_{0}^{i}=v_{i}(t)+\omega_{i}(t)
$$

We get

$$
\left\|S^{\nu}(t) u_{0}^{1}-S^{\nu}(t) u_{0}^{2}\right\|_{0}^{2} \leq 2\left(\left\|v_{1}(t)-v_{2}(t)\right\|_{0}^{2}+\left\|\omega_{1}(t)-\omega_{2}(t)\right\|_{0}^{2}\right)
$$

and

$$
\lim _{t \rightarrow \infty}\left\|v_{1}(t)-v_{2}(t)\right\|_{0}^{2}=0
$$

For any $v \in I$, we have

$$
\begin{aligned}
\frac{d}{d t}\left\|\omega_{1}(t)-\omega_{2}(t)\right\|_{0}^{2} & =2 \int_{\Omega} \nabla\left(\omega_{1}(t)-\omega_{2}(t)\right) \cdot \nabla\left(\partial_{t} \omega_{1}(t)-\partial_{t} \omega_{2}(t)\right) \\
& \leq\left\|\omega_{1}(t)-\omega_{2}(t)\right\|_{0}\left\|\partial_{t} \omega_{1}(t)-\partial_{t} \omega_{2}(t)\right\|_{0} .
\end{aligned}
$$

Now, integrating (3.35) for $t$ from $s$ to $T(0<s<T)$, we have that

$$
\begin{aligned}
& \left\|\omega_{1}(T)-\omega_{2}(T)\right\|_{0}^{2} \\
& \quad \leq\left\|\omega_{1}(s)-\omega_{2}(s)\right\|_{0}^{2}+\int_{s}^{T}\left\|\omega_{1}(t)-\omega_{2}(t)\right\|_{0}\left\|\partial_{t} \omega_{1}(t)-\partial_{t} \omega_{2}(t)\right\|_{0} d t
\end{aligned}
$$




$$
\begin{aligned}
\leq & \left\|\omega_{1}(s)-\omega_{2}(s)\right\|_{0}^{2}+\left[\int_{0}^{T}\left\|\omega_{1}(t)-\omega_{2}(t)\right\|_{0}^{2} d t\right]^{\frac{1}{2}} \\
\times & {\left[\int_{0}^{T}\left\|\partial_{t} \omega_{1}(t)-\partial_{t} \omega_{2}(t)\right\|_{0}^{2} d t\right]^{\frac{1}{2}} }
\end{aligned}
$$

Combining with Lemma 3.9 and Corollary 3.10, integrating (3.36) over $s \in(0, T]$, and dividing $T$ by two sides of inequality, we obtain

$$
\begin{aligned}
\left\|\omega_{1}(T)-\omega_{2}(T)\right\|_{0}^{2} & \leq C_{T} \int_{0}^{T}\left\|\omega_{1}(t)-\omega_{2}(t)\right\|_{0}^{2} d t+\frac{\mathcal{K}_{2}}{T} \\
& =C_{T} \int_{0}^{T}\left\|K^{v}(t) u_{0}^{1}-K^{v}(t) u_{0}^{2}\right\|_{0}^{2} d t+\frac{\mathcal{K}_{2}}{T} \\
& =\varphi_{T}\left(u_{0}^{1}, u_{0}^{2}, v, v\right),
\end{aligned}
$$

where $C_{T}=T / 4+1 / T$.

Combining with Lemma 3.9 and Corollary 3.10, applying Lemma 2.4, then for any $v \in I$ fixed, the sequence $\left\{K^{v}\left(t_{n}\right) u_{0}^{n}\right\}_{n=1}^{\infty}$ is relatively compact in $L^{2}\left(0, T ; H_{0}^{1}(\Omega)\right)$. Then the semigroup $\left\{S^{\nu}(t)\right\}_{t \geq 0}$ is asymptotically compact in $H_{0}^{1}(\Omega)$. Then, obviously, by Theorem 2.2 and letting $v_{n}=v$ for any $n \in \mathbb{N}$, we obtain the global attractor $\mathscr{A}_{v}$, which is invariant, compact in $H_{0}^{1}(\Omega)$, and attracts every bounded subset of $H_{0}^{1}(\Omega)$. Furthermore, by Lemma 3.9,

$$
\mathscr{A}_{\nu} \subset D(A)
$$

holds for any $v>0$. The proof is complete.

\section{Upper semicontinuity in $H_{0}^{1}(\Omega)$}

Lemma 4.1 Letf satisfy (1.4)-(1.5) and $B_{0}$ be the bounded uniformly absorbing set of the semigroup $\left\{S^{\nu}(t)\right\}_{t \geq 0}$ in $\mathcal{V}$ (from Lemma 3.5). Assume that $\left\{v_{n}\right\}_{n=0}^{\infty} \subset I$ with $v_{n} \stackrel{n \rightarrow \infty}{\longrightarrow} v_{0}$ and $x_{n} \stackrel{n \rightarrow \infty}{\longrightarrow} x_{0}$ in $\mathcal{V}$. Then we get

$$
S^{v_{n}}(t) x_{n} \stackrel{n \rightarrow \infty}{\longrightarrow} S^{v_{0}}(t) x_{0} \quad \text { in } L^{2}(\Omega)
$$

holds for all $t>0$ and $v_{0} \in I$.

Proof Let $u^{n}(t), u^{0}(t)$ be the solutions to Eq. (1.1) with the parameters $v=v_{n}, v=v_{0}$ and the initial data $x_{n}, x_{0} \in \mathcal{V}(n=1,2, \ldots)$ respectively, that is,

$$
u^{n}(t)=S^{\nu_{n}}(t) x_{n} \quad \text { and } \quad u^{0}(t)=S^{\nu_{0}} x_{0} .
$$

Setting $\omega_{n}(t)=u^{n}(t)-u^{0}(t)$, then $\omega_{n}(t)$ satisfies the following equations:

$$
\omega_{n t}-\Delta \omega_{n}-v_{0} \Delta \omega_{n t}+f\left(u^{n}\right)-f\left(u^{0}\right)=\left(v_{n}-v_{0}\right) \Delta u_{t}^{n}
$$

The problem is supplemented with the boundary condition

$$
\left.\omega_{n}(x, t)\right|_{\partial \Omega}=0 \quad \text { for all } t \geq 0
$$


and the initial condition

$$
\left.\omega_{n}\right|_{t=0}=x_{n}-x_{0}
$$

Multiplying (4.1) by $\omega_{n}$, integrating in $\Omega$, and arranging, we have

$$
\frac{d}{d t}\left(\left|\omega_{n}\right|_{2}^{2}+v_{0}\left\|\omega_{n}\right\|_{0}^{2}\right)+\left\|\omega_{n}\right\|_{0}^{2} \leq 2 l\left(\left|\omega_{n}\right|_{2}^{2}+v_{n}\left\|\omega_{n}\right\|_{0}^{2}\right)+\left|v_{n}-v_{0}\right|^{2}\left\|u_{t}^{n}\right\|_{0}^{2} .
$$

Using Gronwall's lemma and Lemma 3.7, it follows that

$$
\left|S^{v_{n}}(t) x_{n}-S^{\nu_{0}}(t) x_{0}\right|_{2}^{2} \leq C_{\mathcal{K}_{1}} e^{2 l t}\left(\left\|x_{n}-x_{0}\right\|_{0}^{2}+\left|v_{n}-v_{0}\right|^{2}\right)
$$

Let $n \rightarrow \infty$, we get the desired result immediately. The proof is complete.

Lemma 4.2 Under the assumptions of Lemma 4.1, let $B_{0}$ be the bounded uniformly absorbing set of the family of semigroups $\left\{S^{\nu}(t)\right\}_{t \geq 0}$ in $\mathcal{V}$ (which is from Lemma 3.5). Then, for any $\left\{v_{n}\right\}_{n=1}^{\infty} \subset[0,+\infty),\left\{t_{n}\right\}_{n=1}^{\infty} \subset[0,+\infty),\left\{u_{0}^{n}\right\}_{n=1}^{\infty} \subset B_{0}$, the sequence $\left\{S^{v_{n}}\left(t_{n}\right) u_{0}^{n}\right\}_{n=1}^{\infty}$ is relatively compact in $\mathcal{V}$.

Proof Let $u^{i}(t)=S^{v_{i}}(t) u_{0}^{i}(i=1,2)$ be the solutions to Eq. (1.1) with the parameters $v=v_{i}$ and the initial data $u_{0}^{i}(x) \in B_{0}(i=1,2)$ respectively. By (3.24), $u^{i}(t)=S^{\nu_{i}}(t) u_{0}^{i}=S_{1}^{v_{i}}(t) u_{0}^{i}+$ $K^{v_{i}}(t) u_{0}^{i}=v^{i}(t)+\omega^{i}(t)(i=1,2)$, we get

$$
\left\|S^{\nu_{1}}(t) u_{0}^{1}-S^{\nu_{2}}(t) u_{0}^{2}\right\|_{0}^{2} \leq 2\left(\left\|\nu_{1}-v_{2}\right\|_{0}^{2}+\left\|\omega_{1}-\omega_{2}\right\|_{0}^{2}\right)
$$

and

$$
\lim _{t \rightarrow \infty}\left\|v^{i}(t)\right\|_{0}^{2}=0
$$

Then, for any $\varepsilon>0$, there is $T_{0}$, for any $t \geq T_{0}$ it follows that

$$
\left\|v^{i}(t)\right\|_{0}^{2} \leq \varepsilon / 2 \text { and } \frac{\mathcal{K}_{2}}{4 t} \leq \varepsilon .
$$

Setting $\theta=\omega^{1}(t)-\omega^{2}(t)$, then it follows that

$$
\frac{d}{d t}\|\theta(t)\|_{0}^{2}=\frac{d}{d t} \int_{\Omega} \nabla \theta(t) \cdot \nabla \theta(t)=2 \int_{\Omega} \nabla \theta \cdot \nabla \theta_{t} \leq\|\theta(t)\|_{0}\left\|\theta_{t}(t)\right\|_{0} .
$$

Now integrating (4.6) between $s$ and $T\left(T_{0}<s<T\right)$, we obtain

$$
\begin{aligned}
\|\theta(T)\|_{0}^{2} & \leq\|\theta(s)\|_{0}^{2}+\int_{s}^{T}\|\theta(t)\|_{0}\left\|\theta_{t}(t)\right\|_{0} d t \\
& \leq\|\theta(s)\|_{0}^{2}+\left[\int_{0}^{T}\|\theta(t)\|_{0}^{2} d t\right]^{\frac{1}{2}}\left[\int_{0}^{T}\left\|\theta_{t}(t)\right\|_{0}^{2} d t\right]^{\frac{1}{2}} .
\end{aligned}
$$


Integrating (4.7) over $s \in[0, T]$ and dividing $T$ by two sides of inequality, then

$$
\begin{aligned}
\|\theta(T)\|_{0}^{2} & \leq \frac{1}{T} \int_{0}^{T}\|\theta(s)\|_{0}^{2} d s+\left[\int_{0}^{T}\|\theta(t)\|_{0}^{2} d t\right]^{\frac{1}{2}}\left[\int_{0}^{T}\left\|\theta_{t}(t)\right\|_{0}^{2} d t\right]^{\frac{1}{2}} \\
& \leq\left[\int_{0}^{T}\|\theta(t)\|_{0}^{2} d t\right]^{\frac{1}{2}}\left[\frac{1}{T}\left(\int_{0}^{T}\|\theta(t)\|_{0}^{2} d t\right)^{\frac{1}{2}}+\left(\int_{0}^{T}\left\|\theta_{t}(t)\right\|_{0}^{2} d t\right)^{\frac{1}{2}}\right] \\
& \leq(T+1 / T) \int_{0}^{T}\left\|\omega_{1}(t)-\omega_{2}(t)\right\|_{0}^{2} d t+\frac{\mathcal{K}_{2}}{4 T}
\end{aligned}
$$

and

$$
\begin{aligned}
& \int_{0}^{T}\left\|\omega_{1}(t)-\omega_{2}(t)\right\|_{0}^{2} d t \\
& \quad \leq 2 \int_{0}^{T}\left(\left\|K^{\nu_{1}}(t) u_{0}^{1}-K^{\nu_{1}}(t) u_{0}^{2}\right\|_{0}^{2}+\left\|K^{\nu_{1}}(t) u_{0}^{2}-K^{\nu_{2}}(t) u_{0}^{2}\right\|_{0}^{2}\right) d t \\
& \quad \leq 2 \int_{0}^{T}\left\|K^{\nu_{1}}(t) u_{0}^{1}-K^{\nu_{1}}(t) u_{0}^{2}\right\|_{0}^{2} d t+\left|v_{1}-v_{2}\right| \int_{0}^{T}\left(\left\|\omega_{1 t}(t)\right\|_{0}^{2}+\left\|u_{1 t}(t)\right\|_{0}^{2}\right) d t
\end{aligned}
$$

Then

$$
\left\|\omega_{1}(T)-\omega_{2}(T)\right\|_{0}^{2} \leq \varepsilon+\phi_{T}\left(u_{0}^{1}, u_{0}^{2}, v_{1}, v_{2}\right)
$$

Now we verify that $\phi_{T}\left(u_{0}^{1}, u_{0}^{2}, v_{1}, v_{2}\right)$ is a contractive function on $B_{0} \times B_{0}$. Let $\left\{v_{n}\right\}_{n=1}^{\infty} \subset$ $I$ and $\left\{u_{0}^{n}\right\}_{n=1}^{\infty} \subset B_{0}$ be fixed, combining with Lemma 3.9, Corollary 3.10, and applying Lemma 2.4, then for any $v_{k}$ fixed, the sequence $\left\{K^{v_{k}} u_{0}^{n}\right\}_{n=1}^{\infty}$ is relatively compact in $L^{2}\left(0, T ; H_{0}^{1}(\Omega)\right)$. In light of $I \subset \mathbb{R}^{+}$being a bounded closed set, then $\left\{v_{n}\right\}_{n=1}^{\infty}$ is also relatively compact. From Theorem 2.2, the sequence $\left\{K^{v_{n}} u_{0}^{n}\right\}_{n=1}^{\infty}$ is relatively compact on $L^{2}\left([0, T] ; H_{0}^{1}(\Omega)\right)$. The proof is finished.

Proof of Theorem 1.2 From Theorem 1.1, Lemma 4.1, and Lemma 4.2, we know that the conditions of Theorem 2.3 are all satisfied. The proof is complete.

\section{Acknowledgements}

The authors would like to thank the referees for their many helpful comments and suggestions.

\section{Funding}

The research is financially supported by Hunan Provincial Key Laboratory of Mathematical Modeling and Analysis in Engineering (Changsha University of Science and Technology) and the National Natural Science Foundation of China (Nos. 11101053, 71471020).

\section{Availability of data and materials}

Not applicable.

\section{Competing interests}

The authors declare that they have no competing interests.

Authors' contributions

The first and second authors played a major role in this work. The authors investigated the literature reviews. All the authors read and approved the final manuscript.

\section{Author details}

'School of Mathematics and and Statistics, Changsha University of Science and Technology, Changsha, China. ${ }^{2}$ College of Mathematics and Physics Science, Hu'nan University of Arts and Science, Changde, China. 


\section{Publisher's Note}

Springer Nature remains neutral with regard to jurisdictional claims in published maps and institutional affiliations.

\section{Received: 12 August 2020 Accepted: 26 November 2020 Published online: 28 January 2021}

\section{References}

1. Wang, L., Wang, Y., Qin, Y.: Upper semicontinuity of attractors for nonclassical diffusion equations in $H^{1}\left(\mathbb{R}^{3}\right)$. Appl. Math. Comput. 240, 51-61 (2014)

2. Xie, Y., Li, Y., Zeng, Y.: Uniform attractors for nonclassical diffusion equations with memory. J. Funct. Spaces 2016, Article ID 5340489 (2016)

3. Xie, Y., Li, Q., Zhu, K.: Attractors for nonclassical diffusion equations with arbitrary polynomial growth nonlinearity. Nonlinear Anal. 31, 23-37 (2016)

4. Aifantis, E.C.: On the problem of diffusion in solids. Acta Mech. 37, 265-296 (1980)

5. Barenblatt, G., Zheltov, I.P., Kochina, I.N.: Basic concepts in the theory of seepage of homogeneous liquids in fissured rocks. J. Appl. Math. Mech. 24, 1286-1303 (1960)

6. Chen, P.J., Gurtin, M.E.: On a theory of heat conduction involving two temperatures. Z. Angew. Math. Phys. 19, 614-627 (1968)

7. Peter, J.G., Gurtin, M.E.: On the theory of heat condition involving two temperatures. Z. Angew. Math. Phys. 19, 614-627 (1968)

8. Colton, D.: Pseudo-parabolic equations in one space variable. J. Differ. Equ. 12, 559-565 (1972)

9. Showalter, R.E.: Sobolev equations for nonlinear dispersive systems. Appl. Anal. 7, 297-308 (1978)

10. Sobolev, S.L.: Some new problems in mathematical physics. Izv. Akad. Nauk SSSR, Ser. Mat. 18, 3-50 (1954)

11. Sun, C., Yang, M.: Dynamics of the nonclassical diffusion equations. Asymptot. Anal. 59, 51-81 (2008)

12. Wang, F., Wang, P., Yao, Z.: Approximate controllability of fractional partial differential equation. Adv. Differ. Equ. 2015, 367 (2015)

13. Wang, F., Yao, Z.: Approximate controllability of fractional neutral differential systems with bounded delay. Fixed Point Theory 17(2), 495-508 (2016)

14. Kumar, A., Chauhan, H.V.S., Chokkalingam, R.: Existence of solutions of non-autonomous fractional differential equations with integral impulse condition. Adv. Differ. Equ. 2020, 434 (2020)

15. Valliammal, N., Ravichandran, C., Nisar, K.S.: Solutions to fractional neutral delay differential nonlocal systems. Chaos Solitons Fractals 138, 109912 (2020)

16. Aouadi, M.: Quasi-stability and upper semicontinuity for coupled parabolic equations with memory. Stud. Appl. Math. $145,586-621(2020)$

17. Zhang, J., Xie, Y., Luo, Q., Tang, Z: Asymptotic behavior for the semi-linear reaction diffusion equations with memory. Adv. Differ. Equ. 2019, 510 (2019)

18. Zhu, K., Xie, Y., Zhou, F.: LP-Pullback attractors for non-autonomous reaction-diffusion equations with delays. Topol. Methods Nonlinear Anal. 54(1), 9-27 (2019)

19. Zhu, K., Xie, Y., Zhou, F., Li, X.: Pullback attractors for non-autonomous reaction-diffusion equations in $\mathbb{R}^{n}$. J. Math Phys. 60, 0032702 (2019)

20. Wang, Y., Li, P., Qin, Y.: Upper semicontinuity of uniform attractors for nonclassical diffusion equations. Bound. Value Probl. 2017(1), 84 (2017)

21. Wang, Y., Qin, Y.: Upper semicontinuity of pullback attractors for nonclassical diffusion equations. J. Math. Phys. 51 $022701(2010)$

22. Anh, C., Bao, T.: Pullback attractors for a class of non-autonomous nonclassical diffusion equations. Nonlinear Anal. 73 , 399-412 (2010)

23. Anh, C., Toan, N.: Existence and upper semicontinuity of uniform attractors in $H^{1}\left(\mathbb{R}^{N}\right)$ for nonautonomous nonclassical diffusion equations. Ann. Pol. Math. 111, 271-295 (2014)

24. Zhu, K., Xie, Y., Zhou, F.: Attractors for the nonclassical reaction diffusion equations on time-dependent spaces. Bound. Value Probl. 2020, 95 (2020)

25. Conti, M., Dell'Oro, F., Pata, V.: Nonclassical diffusion with memory lacking instantaneous damping. Commun. Pure Appl. Anal. 19, 2035-2050 (2020)

26. Hu, H., Yi, T., Zou, X.: On spatial-temporal dynamics of a Fisher-KPP equation with a shifting environment. Proc. Am. Math. Soc. 148(1), 213-221 (2020)

27. Hu, H., Tan, Y., Huang, J.: Hopf bifurcation analysis on a delayed reaction-diffusion system modelling the spatial spread of bacterial and viral diseases. Chaos Solitons Fractals 25, 152-162 (2019)

28. Li, J., Liu, F., Feng, L., Turner, I.: A novel finite volume method for the Riesz space distributed-order diffusion equation. Comput. Math. Appl. 74, 772-783 (2017)

29. Ravichandran, C., Logeswari, K., Panda, S.K., Nisar, K.S.: On new approach of fractional derivative by Mittag-Leffler kernel to neutral integro-differential systems with impulsive conditions. Chaos Solitons Fractals 139, 110012 (2020)

30. Panda, S.K., Abdeljawad, T., Ravichandran, C.: Novel fixed point approach to Atangana-Baleanu fractional and $L^{p}$-Fredholm integral equations. Alex. Eng. J. 59, 1959-1970 (2020)

31. Chaudhary, R., Pandey, D.N.: Existence results for a class of impulsive neutral fractional stochastic integro-differential systems with state dependent delay. Stoch. Anal. Appl. 37, 865-892 (2019)

32. Munusamy, K., Ravichandran, C., Nisar, K.S.: Existence of solutions for some functional integrodifferential equations with nonlocal conditions. Math. Methods Appl. Sci. 43, 10319-10331 (2020)

33. Robinson, J.C.: Infinite-Dimensional Dynamical Dystems. Cambridge University Press, Cambridge (2001) 